\title{
Simulation of a 50GeV PWFA Stage
}

\author{
C. Huang ${ }^{1}$, W. Lu ${ }^{1}$, M.M. Zhou ${ }^{1}$, V.K.Decyk ${ }^{1}$, W. B. Mori ${ }^{1}$, E. Oz ${ }^{2}$, C.D.

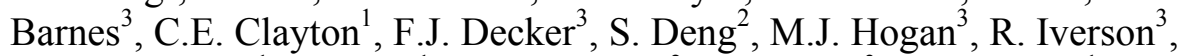 \\ D.K. Johnson ${ }^{1}$, C. Joshi ${ }^{1}$, T. Katsouleas ${ }^{2}$, P. Krejcik ${ }^{3}$, K.A. Marsh ${ }^{1}$, P. \\ Muggli ${ }^{2}$, C. O'Connell ${ }^{3}$, D. Walz ${ }^{3}$ \\ ${ }^{1}$ University of California, Los Angeles 90095 \\ ${ }^{2}$ University of Southern California 90089 \\ ${ }^{3}$ Stanford Linear Accelerator Center 94025
}

\begin{abstract}
The plasma afterburner has been proposed as a possible advanced acceleration scheme for a future linear collider. In this concept, a high energy electron(or positron) drive beam from an existing linac such as the SLC will propagate in a plasma section of density about one order of magnitude lower than the peak beam density. The particle beam generates a strong plasma wave wakefield which has a phase velocity equal to the velocity of the beam and this wakefield can be used to accelerate part of the drive beam or a trailing beam. Several issues such as the efficient transfer of energy and the stable propagation of the particle beam in the plasma are critical to the afterburner concept. We investigate the nonlinear beam-plasma interactions in such scenario using a new 3D particle-in-cell code called QuickPIC. Preliminary simulation results for electron acceleration, beam-loading and hosing instability will be presented.
\end{abstract}

\section{INTRODUCTION}

Plasma wakefield acceleration (PWFA) is one of the advanced acceleration schemes which provides very high acceleration gradients. Since this concept was proposed, many experiments have been implemented to verify the idea and further develop the design into a mulit-dimensional non-linear regime. In the first proof-ofprinciple experiment, $\sim 1 \mathrm{Mev} / \mathrm{m}$ accelerating gradient in the over-dense plasma was observed [1]. While in the recent E164X experiment conducted at SLAC, $3 \mathrm{GeV}$ energy gain over $15 \mathrm{~cm}$ wide plasma section was reported at this workshop [2]. This substantial energy gain is obtained by sending a $30 \mathrm{GeV}$ electron beam into $\mathrm{Li}$ vapor of $1.6 \mathrm{E} 17 \mathrm{~cm}^{-3}$ peak density. The electric field from the beam itself is strong enough to tunnel-ionize the $\mathrm{Li}$ vapor and expel the newborn electrons away from the beam. A nonlinear plasma wakefield is established during this process and the longitudinal acceleration field associates with it is on the order of $10 \mathrm{GeV} / \mathrm{m}$.

An "afterburner" concept based on PWFA has been proposed as an energy booster for an existing linac such as the SLC to double the energy of electron and positron beams before they collide [3]. In this paper, several issues are addressed regarding the efficient transfer of energy from the drive beam to a possible trailing beam and the transverse dynamics and stability of ultra-relativistic beams in under-dense plasmas. To date, linear theory [4] has been used to study the transformer ratio(i.e., $E_{\text {accel }} / \mathrm{E}_{\text {decel }}$, where $E_{\text {accel }}$ and $E_{\text {decel }}$ are the peak acceleration and deceleration fields respectively) 
and the related beam-loading issues. In addition, to date the only theory for the tranverse stability, i.e., the hosing instability, of the drive beam assumes a preformed equilibrium channel [5]. However, our interests primarily focus on the strongly nonlinear and non-equilibrium blow-out regime, namely $n_{b} / n_{0} \gg 1\left(n_{b}\right.$ is the peak beam density and $\mathrm{n}_{0}$ is the ambient plasma density) and a self-formed ion channel case. We have been making theoretical progress on both of these topics, however, to make clear quantitative predictions requires accurate $3 \mathrm{D}$ particle-based computer models. Recently, we have developed a quasi-static parallel PIC code which enables us to conduct the first full scale simulation study of such issues in a $50 \mathrm{GeV}$ afterburner stage. We describe our simulation model in Section II and report on preliminary results in Section III.

\section{QUASI-STATIC PIC MODEL}

In explicit electromagnetic PIC codes, the full set of Maxwell equations are solved to determine the dynamics of finite size macro-particles in their self-consistent electric and magnetic fields. This method includes high frequency modes which are often not important to our problem, causing the time step of the simulation to be severely limited by the Courant condition. For the study of energetic beam transport in a plasma, a non-radiative approach is found to be more convenient. A beam with $\gamma>>1$, , evolves on a time scale corresponding to its betatron period which is $(2 \gamma)^{1 / 2}$ times longer than the plasma period. Thus, we separate out the evolution of the drive beam and the generation of the wake. To illustrate how this is implemented, we start from the Maxwell equations in Lorentz guage,

$$
\begin{aligned}
& \left(\frac{1}{c^{2}} \frac{\partial^{2}}{\partial t^{2}}-\nabla^{2}\right) \phi=4 \pi \rho \\
& \left(\frac{1}{c^{2}} \frac{\partial^{2}}{\partial t^{2}}-\nabla^{2}\right) \mathbf{A}=\frac{4 \pi}{c} \mathbf{j}
\end{aligned}
$$

Next, we rewrite these equations using the $(\mathrm{x}, \mathrm{y}, s, \xi)$ coordinates, where $s=z(z$ is the direction in which the beam is moving), and $\xi=t-z / v_{b}$. We further make the quasi-static approximation, which is essentially done by assuming that $\partial_{s}<<\partial_{\xi}$. Then a set of full quasi-static equations can be written as,

$$
\begin{aligned}
& \nabla_{\perp}^{2} \phi=-4 \pi \rho, \\
& \nabla_{\perp}^{2} \mathbf{A}_{\perp}=-4 \pi \mathbf{j}_{\perp} / c, \\
& \nabla_{\perp} \cdot \mathbf{A}_{\perp}=-\frac{\partial \Psi}{\partial \xi},
\end{aligned}
$$

with the equations of motion being:

$$
\begin{aligned}
\frac{d \mathbf{P}_{b \perp}}{d s} & =q_{b} \nabla_{\perp} \Psi, \\
\frac{d \mathbf{P}_{e \perp}}{d \xi} & =\frac{q_{e}}{1-V_{e / /} / c}\left[\mathbf{E}_{\perp}+\left(\mathbf{V}_{e} \times \mathbf{B}\right)_{\perp}\right],
\end{aligned}
$$


In eqns. (5) and (6), $\Psi=\phi-A_{/ /}$, where $A_{/ /}$is the longitudinal component of vector potential. $q$ is the charge of particle and $\rho$ is the charge density. $\mathbf{V}, \mathbf{P}$ are velocity and momentum and the subscript $\mathrm{b}$ and e denote beam electron and plasma electron respectively.

The transverse evolution is completely decoupled from the longitudinal evolution in eqns. (3) and (4). And in eqns. (6) and (7), the two time scales are clearly separated. The plasma evolves on the fast time scale, $\Delta \xi$, which needs to resolve the plasma frequency $\omega_{p}$, while the beam evolves on a slower time scale, $\Delta s$, which needs to resolve the betatron frequency $\omega_{\beta}=\omega_{p} / \sqrt{2 \gamma}$. Therefore, $\Delta s$ can be two orders of magnitudes larger than $\Delta \xi$ for SLC type parameters.

\section{SIMULATION SETUP}

We describe only one set of parameters. Our simulation uses a moving window which has the same velocity as the drive beam. The beam moves in the negative $\xi$ direction. The simulation parameters are summarized in the following table.

TABLE 1. Simulation parameters.

\begin{tabular}{lc}
\hline Parameters & 50GeV stage simulation \\
\hline Plasma density & $5.66 \mathrm{E} 16 \mathrm{~cm}^{-3}$ \\
Plasma length & $3.06 \mathrm{~m}$ \\
Wavebreaking wakefield & $24 \mathrm{GV} / \mathrm{m}$ \\
Beam charge (drive beam) & $3 \mathrm{E} 10^{10}$ \\
Beam charge (trailing beam) & $1 \mathrm{E} 10^{10}$ \\
Beam separation & $111 \mu \mathrm{m}=5 \mathrm{c} / \omega_{\mathrm{p}}$ \\
Beam emittance & $2230 \mathrm{~mm} \cdot \mathrm{mrad}$ \\
Beam initial energy & $50 \mathrm{GeV}$ \\
Longitudinal beam profile (drive beam) & wedge shape, \\
& $\mathrm{L}=145 \mu \mathrm{m}=6.5 \mathrm{c} / \omega_{\mathrm{p}}$ \\
Longitudinal beam profile (trailing beam) & $\mathrm{Gaussian} \mathrm{shape,}$ \\
& $\sigma_{\mathrm{z}}=10 \mu \mathrm{m}=0.45 \mathrm{c} / \omega_{\mathrm{p}}$ \\
Transverse beam profile (drive beam) & $\mathrm{Gaussian} \mathrm{shape,}$ \\
& $\sigma_{\mathrm{r}}=15 \mu \mathrm{m}=0.67 \mathrm{c} / \omega_{\mathrm{p}}$ \\
Transverse beam profile (trailing beam) & $\mathrm{Gaussian} \mathrm{shape,}$ \\
& $\sigma_{\mathrm{r}}=10 \mu \mathrm{m}=0.45 \mathrm{c} / \omega_{\mathrm{p}}$ \\
Simulation cell size & $0.05 \times 0.05 \times 0.066 \mathrm{c} / \omega_{\mathrm{p}}$ \\
Number of cells & $256 \times 256 \times 256 \mathrm{cells}$ \\
\# of simulation beam particles & $8 \mathrm{million}$ \\
\# of simulation plasma particles in 2D & 262,144 \\
\hline
\end{tabular}

In this preliminary study we confined our investigation to studying the energy gain of the trailing beam and the possible hosing growth from the intense beam-plasma interaction. The size of this simulation represents a huge challenge for explicit PIC code, even on today's state-of-art massive parallel computer it is not possible to conduct a full simulation of such a $50 \mathrm{GeV}$ afterburner stage. Such a simulation would 
take 250,000 node hours using a fully explicit code such as OSIRIS; however, using QuickPIC, our simulation requires only about 2,500 node hours.

\section{SIMULATION RESULT}

As the drive beam (it is located from $-6.5 \mathrm{c} / \omega_{\mathrm{p}}$ to 0 in fig. 1 , and moves to the left), generates the plasma wave wakefield, it loses energy. So the wakefield at the front of the beam is a decelerating field, which is positive in fig. 1. However, the drive beam we used is a wedge shape in the axial direction. For such a longitudinal profile the plasma electrons are blown out adiabatically, so the drive beam transfers energy into plasma wake at a constant rate throughout the beam, i.e., the initial decelerating fields, which is the solid curve in fig. 1 , is flat from $-3 \mathrm{c} / \omega_{\mathrm{p}}$ to 0 . The trailing beam is placed at $5 \mathrm{c} / \omega_{\mathrm{p}}$ behind the first beam. As can be seen from fig. 1 , the acceleration field is relatively flat over the region where the trailing beam exists (In linear wakefield regime, this would be a superposition of the wakes formed by the two beams, however, in blow-out regime it is no longer true. In some cases, the wakefield in this region can be very flat). The initial transformer ratio is about 2 . The dashed curve shows the wakefield at the end of the simulation. The first wake structure slips backward and shortens; however the second one where trailing beam exists doesn't slip, which causes the beam-loaded transformer ratio to decrease. The slippage is due to the etching of the beam head where the focusing ion channel is not fully formed. The beam head diverges because of the large emittance, and at the end of the simulation the beam becomes trumpet-shape. We chose the large emittance so that the bulk of the beam would be matched and the spot size of the beam would not be smaller than a cell size. This simulation was intended to demonstrate that it was possible to double the energy of a high-energy beam with ultra-high gradients. In the future, we will use emittances for the trailing beam that will be of interest to a future collider.

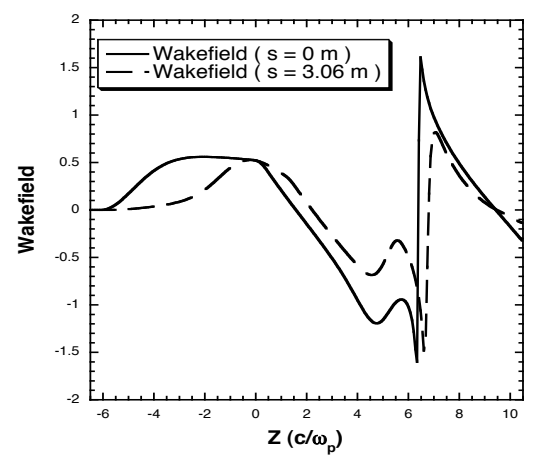

FIGURE 1. The wakefield $e E_{/ /} /\left(m c \omega_{p}\right)$ is plotted as the drive beam enters the plasma and after 3.06 meters of propagation.

The trailing beam is able to gain $50 \mathrm{GeV}$ over $3.06 \mathrm{~m}$ as can be seen from the phase space plot in fig. 2. The acceleration gradient is not constant during the 3.06 meters of propagation, it dropped from $\sim 26.5 \mathrm{GV} / \mathrm{m}$ to $\sim 14.4 \mathrm{GV} / \mathrm{m}$ during the simulation. 
However the acceleration region stays close to the trailing beam center, so a large portion of the beam particles can witness the strong wakefield. This is important because a high quality mono-energetic beam is required in any future linear collider. This preliminary study reveals that the trailing beam preserves its transverse emittance well.

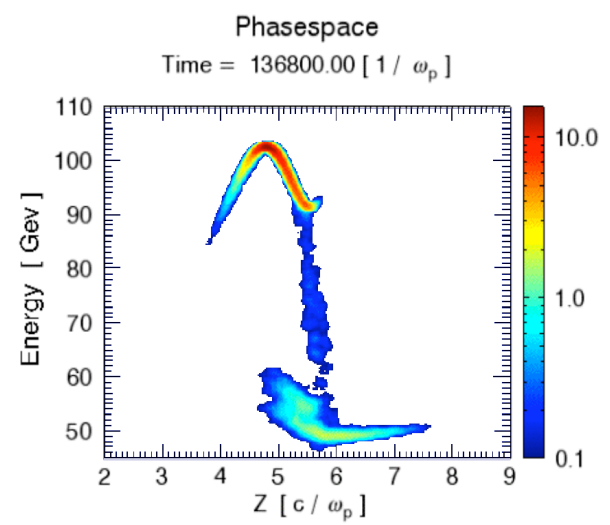

FIGURE 2. Trailing beam phase space at $\mathrm{s}=3.06 \mathrm{~m}$.

In fig. 3 we show the energy distribution of the trailing beam. Two peaks, one at the initial energy, the other one centered around $100 \mathrm{GeV}$ are clearly observed. The first peak accounts for about $30 \%$ of the particles, and the second peak has about $70 \%$ of all the particles. There are very few particles between these two peaks. So two beams are actually formed with a distinct energy difference between them. The accelerated beam also have two peaks and the main peak has an energy spread of $6 \%$ which is promising and may be improved by fine-tuning the initial parameters.

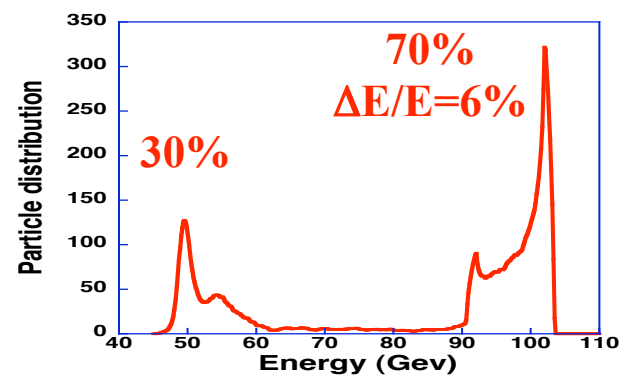

FIGURE 3. Particle energy distribution of the trailing beam at $\mathrm{s}=3.06 \mathrm{~m}$.

Hosing growth which leads to unstable beam transportation could be catastrophic to PWFA. According to linear hosing theory for a preformed equilibrium ion channel, 
one would predict huge hosing growth after the approximate 50 betatron oscillations in the simulation. However, there is almost no hosing observed in the simulation for the wedge-shape drive beam (Fig. 4 solid curve), while small amount of hosing does occur in the trailing beam. This is shown in fig. 4.

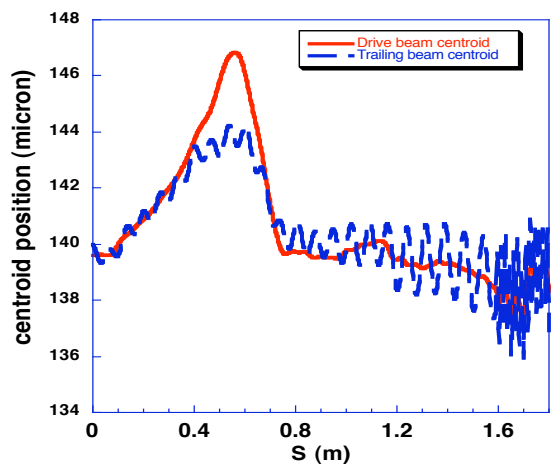

FIGURE 4. Particle energy distribution of the trailing beam at $\mathrm{s}=3.06 \mathrm{~m}$.

In our simulation, the beam particles are generated by a non-uniform random number generator. So although there is no beam head-tail offset(tilt) in this simulation, the noise associated with the particle initialization process is large enough to trigger the hosing instability. In fact, numerical integration of the coupled beam and channel centroid equations shows that even a small amount of noise in the beam centroid can cause similar hosing growth comparable to the tilted beam centroid case. Thus, we believe that the reduced growth rate in the simulation is not due to the difference in the initial conditions. The simulation indeed represents a regime with less hosing growth rate because the existing hosing analysis does not apply. Further theoretical analysis and simulation are required to study this in more detail.

\section{CONCLUSION}

A parallelized quasi-static code called QuickPIC for modeling PWFA was described. QuickPIC reduces the computation need of PWFA simulations and enables us to conduct the first 3D simulation study for a $50 \mathrm{GeV}$ PWFA afterburner stage. Results from this study show that $50 \mathrm{GeV}$ energy gain can be obtained in a $3 \mathrm{~m}$ long PWFA section while maintaining good beam quality. Little hosing instability is observed and it suggests that a new regime with reduced hosing growth rate may exist.

\section{ACKNOWLEDGMENTS}

This work is supported by the US Department of Energy through grants DE-FC0201ER41179, DE-FG02-3ER54721, DE-FG03-92-ER4727 and ILSA at LLNL. 
Simulations are carried out at NERSC. The authors would also like to thank J. H. Cooley and T. M. Antonsen of University of Maryland for useful discussions and helps for developing QuickPIC.

\section{REFERENCES}

1. J.B. Rosenzweig, et. al., Phys. Rev. Lett., Vol. 61, 98 (1988).

2. see this Proceedings.

3. S. Lee, et. al., Phys. Rev. STAB, Vol. 5, 011001 (2002).

4. P. Chen, et. al., Phys. Rev. Lett., Vol. 54, 693 (1985).

5. D. H. Whittum, et. al., Phys. Rev. Lett., Vol. 67, 991 (1991). 